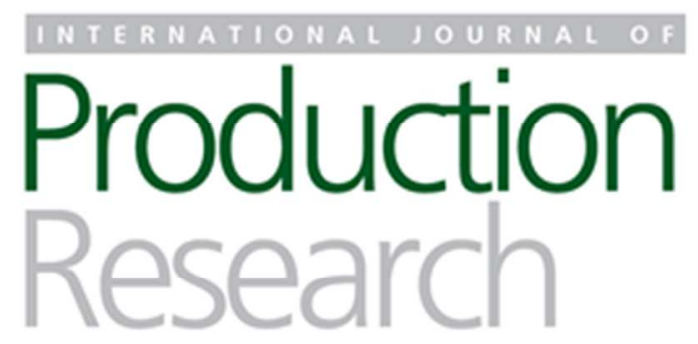

Official Journal of the International foundation for Production Research

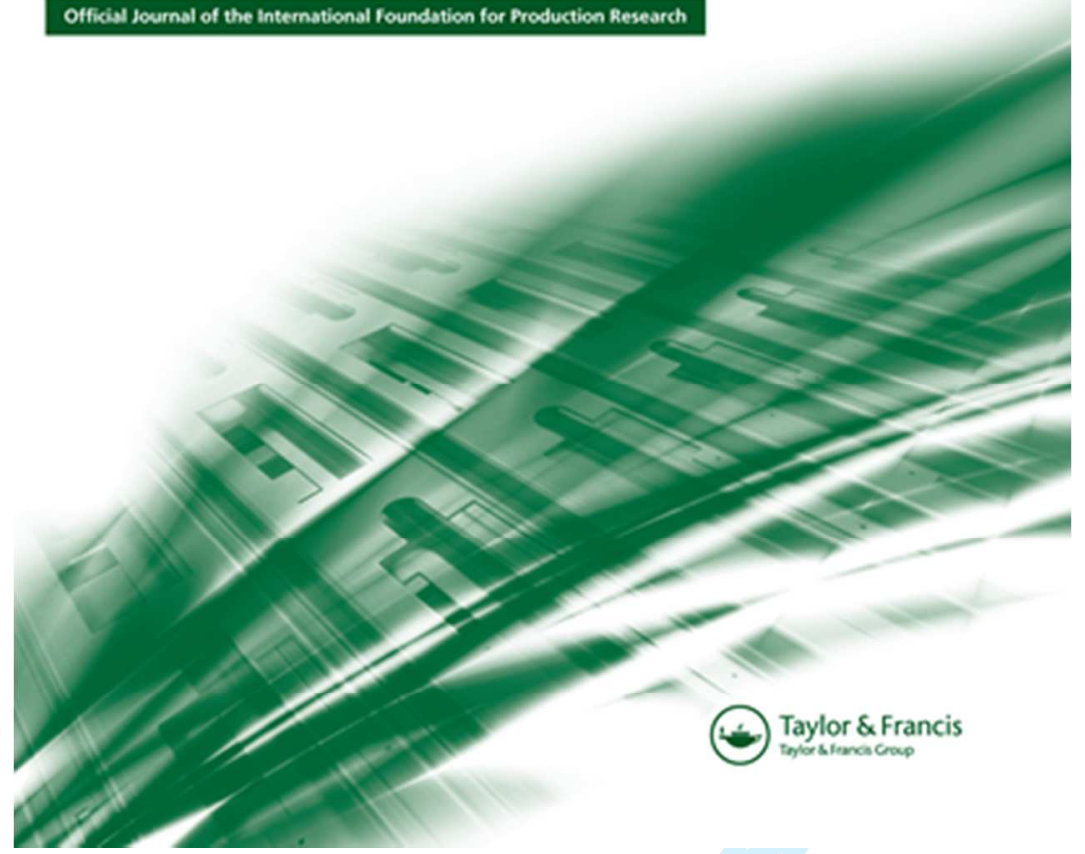

Methodological elements of supply chain design

\begin{tabular}{|r|l|}
\hline Journal: & International Journal of Production Research \\
\hline Manuscript ID: & TPRS-2013-IJPR-1801.R3 \\
\hline Manuscript Type: & Original Manuscript \\
\hline Date Submitted by the Author: & $\mathrm{n} / \mathrm{a}$ \\
\hline Complete List of Authors: & $\begin{array}{l}\text { Corominas, Albert; Universitat Politécnica de Catalunya, Institute of } \\
\text { Industrial and Control Engineering } \\
\text { Mateo, Manel; Universitat Politècnica de Catalunya, Department of } \\
\text { Management } \\
\text { Ribas, Imma; Univeratitat Politecnica de Catalunya. BarcelonaTech, }\end{array}$ \\
\hline
\end{tabular}



Rubio, Sergio; Universidad de Extremadura, of Business Management \& Sociology 


\title{
Methodological Elements of supply chain design
}

\author{
Albert Corominas ${ }^{1,2}$, Manel Mateo ${ }^{2}$, Imma Ribas ${ }^{21}$, Sergio Rubio ${ }^{3}$ \\ ${ }^{1}$ Institute of Industrial and Control Engineering, Universitat Politècnica de Catalunya, \\ Spain \\ ${ }^{2}$ Department of Management, Universitat Politècnica de Catalunya, Spain \\ ${ }^{3}$ Department of Business Management \& Sociology, Universidad de Extremadura, \\ Spain
}

\footnotetext{
${ }^{1}$ Corresponding author: Imma Ribas Avda. Diagonal 647, H7 08028 Barcelona Phone: +34934016587 Fax: +34934016054

E-mail address: imma.ribas@upc.edu (I. Ribas) albert.corominas@upc.edu (A. Corominas) manel.mateo@upc.edu (M. Mateo) srubio@unex.es (S.Rubio)
} 


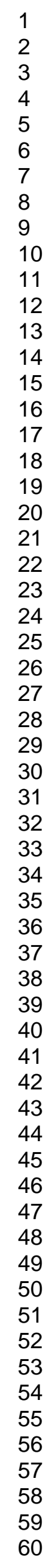

37 


\title{
Methodological Elements of Supply Chain Design
}

\begin{abstract}
After a discussion of the methodological proposals for supply chain design published so far, we describe a hierarchical method consisting of five stages, as well as related concepts and tools. The aim of this method, named SCOP (Supply Chain Outline Process), is to provide a frame for the decision-making about the supply chain configuration. The approach is applicable to all types of supply chains.
\end{abstract}

Keywords: Supply chain design, supply chain network.

\section{Introduction}

In recent years, the concept of supply chain has gained momentum both in academia and in business (Stock and Boyer, 2009; Corominas, 2013). The supply chain (hereinafter, SC) is a network whose nodes are entities that cooperate to provide customers and users with products, whether goods or services, and, where appropriate, to recover, treat and maybe reuse products fully or partially. The word "network" is key because it means that most SCs have a more complex structure than a chain, and that flows between entities are an inseparable part of the SC. The management of SCs poses new problems that require their own methods and tools.

The view of SC as a set of agents that cooperate to achieve shared goals optimally may lead us to forget that, in fact, in each SC there is a leading entity (company, public institution, NGO, association, club, foundation, etc.) that sets the objectives of its SC and, to a certain extent, has the authority to decide who forms part of it and who does not (of ourse, the leading entity has the power to decide its suppliers, but it might not have that of deciding the suppliers of its suppliers; in this case, the suppliers' suppliers, although contributing to the SC, may be considered outside its frontiers). In fact, the leadership is considered the third building block in the house of SCM (Stadtler, 2002), and according to Stadtler and Kilger (2005) there can exist a 'natural' leader or a steering committee. The leading entity (also called dominating unit -Chandra and Grabis, 2007- or focal firm -Wagner and Neshat, 2010-) is the SC nucleus, driver or leader. It is significant that most works on SC refer to its objectives as a whole and do not highlight the critical role of the leading organisation.

SC design is a strategic problem, whose solution strongly influences the performance of the SC, that comprises the decisions regarding the number and location of production facilities, the amount of capacity at each facility, the assignment of each market region to one or more locations, and supplier selection for sub-assemblies, components and materials (Chopra and Meindl, 2012). Chandra and Grabis (2007) define SC configuration as the determination of the size and location of the units to be included in the SC and the links among them), what coincides substantially with the definition of SC design by Chopra and Meindl (2012). The complexity of the SC design is illustrated in Klibi et al. (2010), where some strategic questions to be answered in the design process are formulated: Which markets should we target? What delivery time should we provide in different product markets and at what price? How many production and distribution centres should be implemented and where should they be located? Which activities should be externalized? Which partners should we select? What production, storage and handling technologies should we adopt and how much capacity should we have? Which products should be produced / stocked in each location? Which factory / DC [distribution centre] / demand zones should be supplied by each supplier/ factory/ DC? What means of transportation should be used? In the same paper it is pointed out that the activities of concern include those of recovery and revalorisation. 
Although most early research on SC focused on the management of a given configuration, current literature on SC design has many significant contributions. Some of these works discuss general concepts of design approaches but most propose mathematical programming models, sometimes of great complexity and considerable size. However, the SC design problem cannot be reduced to the formulation and solution of a model since no model can incorporate the multitude of qualitative and quantitative decisions necessary to determine a SC configuration. Even in the simplest cases, there are previous and subsequent decisions to the use of the model.

Given that SC design is usually a complex, lengthy process, it is advisable to follow a method, understood as a series of ordered steps with guidelines and tools to facilitate their implementation (Chandra and Grabis, 2007).

The contribution of this paper is to propose a methodology for the supply chain design that formalises the decisions that should be taken during the design process. The methodology leads to a hierarchical top-down decision process in order to consider all relevant aspects of the SC design. An example involving a real-life problem illustrates the use of the method.

The remainder of this paper is organised as follows. Section 2 summarises the published methodological contributions most relevant to our view on SC design. In section 3 we outline the proposed method. An illustrative example is shown in section 4. Finally, section 5 contains the conclusions and proposes future lines of research.

\section{A summary of the methodological contributions in the SC design literature}

Although numerous publications deal with SC design, few refer more or less explicitly to the methodology.

One of the first approaches is to consider the relation between the type of product and the type of its SC. Fisher (1997) proposes the distinction between efficient and responsive SCs, reintroduced, with a change in the terminology, and extended by Vonderembse et al. (2006). Other contributions about the relations between product design and SC design can be found in Appelqvist et al. (2004), Lamothe et al. (2006), Seuring (2009), Chiu and Okudan (2011) and Marsillac and Roh (2014).

Other authors (Wang et al., 2004; Sha and Che, 2006) focus on the supplier selection, viewed as the central question in the SC design.

However, most papers dealing with SC design tend to identify the design process with the formulation and resolution of a mathematical programming model. Usually, the structure of the SC is represented by means of a multistage graph (e.g., Balinski, 1965; Geoffrion and Graves, 1974; Simchi-Levi et al., 2008; and Chopra and Meindl, 2012) or a network, i.e., a directed graph without any special structure (e.g., Melo et al., 2005; and Laínez et al., 2009). This serves as a starting point to formulate a mathematical programming model (usually a mixed integer linear program). The model can be static or dynamic, deterministic or stochastic. It may admit the possibility of opening new facilities only or closing existing ones as well. Revisions of the model-based literature are found, e.g., in Beamon (1998), Goetschalckx et al. (2002), Meixell and Gargeya (2005) and Shen (2007). Other works propose mathematical models for the SC design on specific industries. 
Finally, a relatively reduced number of papers consider directly methodological aspects.

One of the most relevant is Lakhal et al. (1999), which focuses on the concept of product-market chain (hereafter P-MC). Every product has its own P-MC, represented by the graph of activities, in which specific symbols are used. According to Carle et al. (2012), models based on the graph of activities were subsequently proposed by Vila et al. (2006) and M'Barek et al. (2010). Four basic components must be defined for each P-MC: elementary durable resources, elementary methods, elementary activities and products (include the inputs, outputs and intermediary, and may be material or not, like information, knowledge or services). "Elementary" means here that corresponds to the highest level of detail required. Three types of activities are considered: time, which consists in preserving something for some time; space, representing a change of position in space; and form (assembly operations, order processing or decision making). As the graph of a P-MC is a very detailed representation of the processes that take place in it, its definition requires a deep analysis of these processes. Once the graph of the P-MC, the attributes of the components, the functions associated with each method and the objectives are defined, it is relatively straightforward to formulate a mathematical program to optimise results. Lakhal et al. (1999) give only very general indications to this respect; however, Lakhal et al. (2001) formulate a static model from the graph of activities and propose a heuristic to solve it.

In Vila et al. (2006) the graph of activities is applied to the timber industry. The authors present a complex dynamic model and its resolution by CPLEX and a list of five steps in SC design, particularly interesting from a methodological point of view: definition of the product-markets, sourcing context and planning horizon; definition of product families and the elaboration of the manufacturing-storage activities process graph; definition of potential network resources and technology dependent recipes for production activities; definition of the revenues and costs associated to the network design and activity decisions; optimal mapping of the process graph onto the potential network resources.

Carle et al. (2012), also within the framework based on the graph of activities, propose a dynamic model and a classification of activities somewhat different from that in Lakhal et al. (1999): fabrication-assembly, warehousing-storage and consolidationtranshipment. As in Lakhal et al. (1999), the decision whether an activity is internal or external is not based on the model, but established a priori.

The approach proposed in Lakhal et al. (1999) and complemented by Vila et al. (2006) and others provides a good methodological basis. However, in our view, it obliges to consider all the details from the beginning. This might be appropriate for a very simple SC, but probably not for most SCs. We believe that a hierarchical top-down process is more suitable to address the complexity of network design. The consideration of details without the context of an overview tends to inhibit the generation of alternative proposals, and probably leads to the unnecessary consideration of too many elements in an explicit way. Certainly, the discussed approach seems to favour premature decision making, such as separation of external and internal activities or location of activities. Note that transport is carried out between activity points and transport from one activity to another can only be quantified if their respective locations have been previously determined.

For their part, Talluri and Baker (2002) propose a mathematical programming approach in which two phases (identification of business processes types and potential candidates; evaluation and selection of the units) precedes the formulation and resolution of the model. The methodology described in Dotoli et al. (2005) consists of 
three phases (candidate selection, network design -using a mathematical programming model- and evaluation and validation).

Chandra and Grabis (2007) offer a literature review about SC configuration, including ninety-one papers published up to 2006. The authors themselves propose a methodology that includes eleven steps, embracing all the decision processes from the initiation of the configuration initiative to the evaluation of the configuration decisions, with an emphasis on the information requirements (the step that is more specific to the SC design is the third one, "Information modeling").

Another approach is the Supply Chain Operations Reference (SCOR) model, developed by an independent non-profit organization, the Supply Chain Council (SCC). It is a reference model as a diagnostic tool for the SCM, but without possibilities to do dynamic analysis (Long, 2014). However, its objective is not specifically the process of designing a SC and the complete information about SCOR is available only to the SCC members.

In the process of designing a SC it is to be taken into account that, because of their complexity, SCs have a strong propensity to be disturbed by various natural or nonnatural factors. Therefore, a distinction between uncertainty and risk is necessary (Klibi et al., 2010; Klibi and Martel, 2012). We associate uncertainty with the possibility of realisation of one or another scenario (e.g., relative to demand), and risk with the disruptions caused by quality or delivery terms failures in the supply of raw materials or components, natural disasters, attacks, inclement weather, or traffic accidents among others. Scenarios have been extensively studied; therefore, there is abundant literature available (e.g., Santoso et al., 2005 and Klibi and Martel, 2012). For a deeper consideration of risk in the SC: Tang and Musa (2011), a literature survey on SC Risk Management (SCRM); Micheli et al. (2014), about the selection of mitigation measures and Araújo et al. (2014), a proposal of classifying SC risks. In Klibi et al. (2010), several definitions of robustness, responsiveness and resilience are reviewed and the importance of these concepts in SC design is discussed. Vlajic et al. (2012) associate robustness with the ability to resist disruptions and resilience with the capacity to recover after disruptions, classify possible disturbances and propose redesign principles and strategies for robust behaviour of food SC

Summing up, although valuable methodological elements can be found in the literature, our analysis reveals that there is no sufficiently detailed, complete proposal of a method to guide the design of a SC.

\section{An outline of the method: SCOP (Supply Chain Outline Process)}

Leukel and Sugumaran (2013) point out the two main difficulties faced by SC design. The first one is that "the design space contains a vast number of alternatives, which makes it hard for designers to evaluate and select the best alternative". The second is that "requires sharing and understanding design information by various parties". Our purpose is to contribute to mitigate these difficulties with a method that we call SCOP (Supply Chain Outline Process). SCOP may help throughout the design process, avoiding the premature discarding of some options and sparing the detailed consideration of others as well. Moreover, SCOP facilitates the communication and is a starting point to develop a terminology and a grammar (Leukel and Sugumaran, 2013) in order to avoid ambiguities and make easier checking the correctness of the models. The main characteristics of SCOP are the following: 
a.- Hierarchical top-down approach, avoiding the consideration from the outset of unnecessary details and the premature adoption of decisions. Particularly, the decisions about the activities are considered before those concerning the actors, what may reduce significantly the analysis of alternatives.

b.- The structure of the SC is not supposed to be a priori a tiered one. Any kind of relations between the actors may be considered.

c.- Reverse logistics is considered from the first stage of the method.

d.- A list of alternatives, with indications about the circumstances in which it would be advisable to adopt each one of them, is provided for every step of the decision process.

e.- The activities are classified according to the diverse types and modes of relations between inputs and outputs, so a pair type-mode is assigned to each activity.

f.- The possibility that a significant delay may exist between the beginning of an activity and the availability of its outputs is taken into account explicitly.

g.- In case the decision maker retains more than one option for one or more activities, the method can lead to different solutions to be analysed considering the adopted criteria.

h.- The proposed graphs are tools to represent in a precise way the relations between activities and between actors. Moreover, they may make easier the formulation of the mathematical models.

i.- SCOP is an open frame, which can be deepened (for instance, enlarging the number of distribution options) without changing its basic structure.

Some of these characteristics are present in other methodological proposals concerning SC design. However, to the best of our knowledge none offers all them together.

In Figure 1 an overview of the proposed method is given (stages are described below). The term "product" is used to refer to a single product or family of products, without consideration of the peculiarities of each case. The whole method can be seen as a help to answer the questions formulated in Klibi et al. (2010), synthesised in Section 2. As in any hierarchical decision process, decisions taken at a particular level can force or make desirable to review previous decisions taken at higher levels.

Figure 1.General layout of SCOP

Although a chain is represented in Figure 1 (each link is a stage in the process), this reflects only the case in which a unique alternative is retained at every stage. Otherwise, the right representation would be a directed tree. The decision maker can retain several alternatives for the macrostructure and each one may generate diverse mesostructure configurations (however, most likely, one microstructure will derive from a given mesostructure).

According to Figure 1, one or more graphs (with the corresponding vertexes and the links between them) should be defined in stages 2, 3 and 4 of the method. However, 
we do not claim that it is always necessary to draw the graphs, although we think that the graphical representations will frequently be useful to show concisely the corresponding structures and as a communication tool within the design team.

\subsection{Stage 1: definition of the object, analysis of the environment and determination of the objectives}

The design process must obviously start with the definition of the SC object. "Object" seems more appropriate than "product" or "mission". For instance, the objects of a SC relating to potatoes for consumption may be to grow and sell potatoes to large food retail chains; buy them from large producers and sell them to large retail chains... The definition of the object must include a definition of the product, but this is not enough. Potatoes may be for consumption or for seed potatoes. To give another example, decisions on the distribution types (via retailers or the Internet) can radically affect the nature of businesses and candidate markets.

The definition of the object of the SC ought often to include a characterization of the potential markets for the product. This delimitation of the potential markets may have significant implications on some treats of the SC (e.g., on the distribution options or pricing policies).

The definition of the SC object usually has a strong influence on its design, including the elimination of many a priori options. For example, the Internet sale of audiovisual materials as SC object excludes other ways of distribution.

Once defined the object of the SC, one can proceed to analyse the SC environment (SC Ecosystem, Viswanadham and Kameshwaran, 2013; the influencers, Melnyk et al., 2014). In fact, this analysis may interact with the definition of the object of the SC. A particularly relevant derivation is the definition of the scenarios to be considered (in very complex settings, the use of simulation can help to define the most relevant scenarios).

Generally, objectives are subordinated to the SC object and may include, among others, maximisation of the stock market value of shares, profit, reliability, robustness and resilience; cost or response time minimisation; or covering a set of markets as soon as possible. At this stage, a set of objectives must be established and their relationships, if possible (otherwise, at later stages). The planning horizon should be included.

\subsection{Stage 2: definition of the SC macrostructure}

At this point, the presence of large blocks as part of the SC and the relationships between them are defined. The result may be represented in the called M-graph.

All SC activities involve procurement, production and distribution, and a set of users (often properly called markets or customers). Some of these activities can be insignificant, but they are always somehow present. Thus, we will always have a procurement-production-distribution block, $\mathrm{P}$, and a block representing all users. The simplest SC macrostructure is therefore represented by the M-graph A in Figure 2 and corresponds to a SC without reverse logistics. Waste generated by procurement, production and distribution activities might be sent to what we call depots (block D), which may be landfills, waste treatment plants, sorting centres for further processing or charitable organisations (M-graph B, Figure 2). 
Figure 2. Examples of M-graphs

It is difficult to find examples of SC without the more or less important presence of reverse logistics. Sometimes the management of returns requires a significant logistics effort. The SC may include collection of obsolete products from some collection points (block $\mathrm{C}$ ); therefore, either block $\mathrm{D}$ and/or a set of remanufacturing or recycling activities (block R) must be in the SC. Block $S$ (for secondary markets) is for remanufactured products sold in specific markets. Without any claim to completeness, these blocks in different SC macrostructure configurations are shown in the M-graph $\mathrm{C}$ (Figure 2).

The main consideration in reverse logistics network design is the choice between an independent network for the collection of end of use (EoU) products and a network integrated into the forward SC, resulting in a closed-loop supply chain (CLSC). Akçali, et al. (2009) note that network design (ND) for reverse supply chain "is concerned with establishing an infrastructure to manage the reverse channel only" and in ND for CLSC there is "an infrastructure to manage both the forward and reverse channels in a coordinated manner". In any case, both are set around two critical activities: (i) collection of EoU products and (ii) recovery of their economic value (remanufacturing, reusing and recycling). In the literature on reverse logistics most of the authors propose a four-step reverse logistics system (Lambert et al., 2011): gatekeeping, collection, sorting and disposal. In our case, collection and reprocessing (usually including sorting) are described as a part of the SC mesostructure, meanwhile disposal is a block belonging to the macrostructure previously defined.

\subsection{Stage 3: definition of the SC mesostructure}

The definition of mesostructure requires a sufficiently defined product structure (modules or subassemblies) and production process.

SC mesostructure corresponds to the relations between the classes of elements contained in each vertex of the M-graph, where a class is the set of elements (which are not necessarily and probably not identified at this stage) capable of executing a particular recipe, i.e., a transformation of inputs into outputs. The mesostructure is represented by the called $m$-graph, where each class corresponds to a vertex or cluster of vertices connected with other classes, inputs and outputs of the class in question being outputs and inputs of those classes.

The m-graph is a tool to represent in a compact, precise way the set of activities to be performed in the SC and their relations. The $\mu$-graph (see 3.4.1), which derives from the m-graph, includes all the information required to formulate, if necessary, a mathematical programming model.

The consideration of recipe depends on the point of view, since a recipe can often be decomposed into smaller recipes. As a general orientation, it may be considered as a recipe the whole transformation processes in the same facility.

Generally, the transformation of an M-graph vertex into the corresponding set of $\mathrm{m}$ graph vertices may have diverse variants, some of which are incompatible. Some can be ruled out by what we call strategic decisions; others must be evaluated separately.

From the knowledge about the product and process, one must decide which options to consider in detail regarding: 
- Procurement and production

- Distribution

- Collection

- Reprocessing

The literature has addressed these groups of activities in several levels of detail (see Subsection 3.3.1). In each group, classes may be of various types, according to the relationship between inputs and outputs.

\subsubsection{On the options}

\section{Procurement and production options}

The most important alternative for each recipe is to buy or make, i.e., entrusting an external entity with its execution or executing it in-house. Since the former admits variants, the options to consider are:

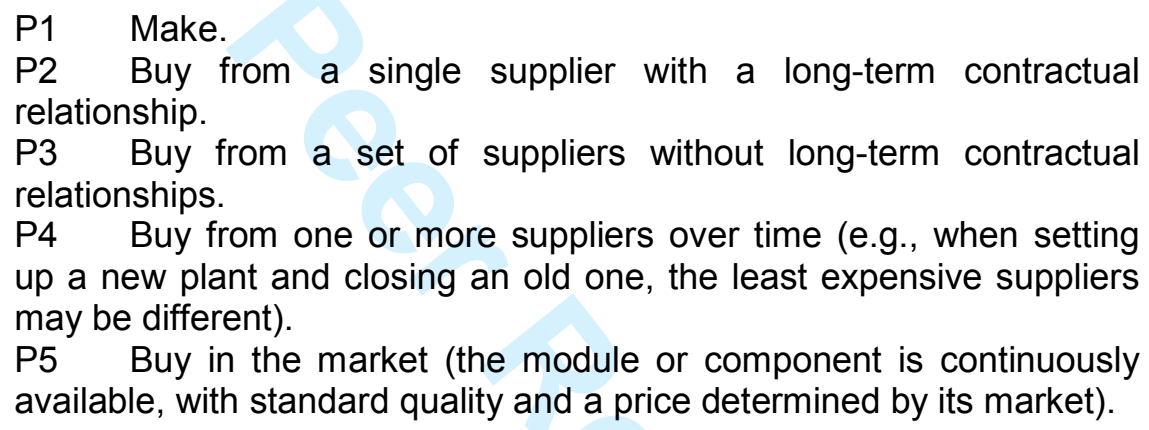

"Make" (P1) must be chosen when the recipe requires the leading entity's expertise that gives a specific character to the product and the company does not want to take risks regarding the dissemination of such knowledge. At the other end, an alwaysavailable component of consistent quality will be purchased in the market (P5).

Decisions about in-between options are more complex. Long-term commitment with a single supplier (P2) offers a cost reduction for both parties, but implies the risk of failure of the supplier and a somewhat difficult situation (Inman and Blumenfeld, 2014). P3 involves a lower cost reduction in exchange for an increase in reliability.

Shortly, the selection criteria for the option for each recipe are confidentiality, reliability (concerning quality and delivery time) and cost.

The degree of product modularity and the selection of options in this group are interdependent. According to Ülkü and Schmidt (2011), product architecture is the key to move from option P1 (integrated chain) to the rest, i.e. decentralised chain.

The choice among the five options depends heavily on the degree of modularity of the product. The decomposition into parts reduces design complexity. In production, common modules and a manufacturing process with a hierarchy of assembly steps simplifies the flows, provides flexibility against uncertainty in demand and reduces inventories (Langlois and Robertson, 1992; Ulrich, 1995). Finally, modularity facilitates product configuration, updating and maintenance by the user (Ramachandran and Krishnan, 2008).

Distribution options 
An extensive and excellent discussion of distribution network design for manufactured products can be found in Chopra and Meindl (2012).

They consider options D1 to D6 in the following list, to which we add D7.

D1 Manufacturer storage with direct shipping to customer.

D2 Manufacturer storage with direct shipping and in-transit merge of components of the order in intermediary facilities.

D3 Distributor storage and home delivery by a logistic operator.

D4 Distributor storage and home delivery with last-mile delivery.

D5 Manufacturer or distributor storage with customer pickup.

D6 Retail storage with customer pickup.

D7 Centralised storage with customer pickup at a retailer under previous order.

D5 and D6 differ in that in the former customers must travel to product storage facilities, but in D6 most customers can find a store within walking distance from their homes. The choice between D1 and D7 depends on the characteristics of the product and the density of the pickup retailer network in D7.

Chopra and Meindl (2012) describe in detail, illustrate with examples, assess options D1 to D6 against cost and service criteria and draw conclusions about the suitability of each option to different product features.

\section{Collection options}

Alumur et al. (2012) highlight that reverse logistics network configuration is a complex problem that requires the determination of optimal localisations and capacities of collection centres, sorting centres, remanufacturing facilities and/or recycling plants.

The return process for EoU products begins with their collection, for which the following options are considered:
C1 Directly by manufacturer or remanufacturer
C2 Through a network of distributors and retailers
C3 Through third-party logistics providers (3PL)

The residual value of the EoU products and their marginal value of time (MVT; Blackburn et al., 2004) can be considered as the selection criteria for the most appropriate collection option. Option $\mathrm{C} 1$ with a pick-up system is generally used to collect EoU products with high residual value and high MVT (e.g., computers); C2 with a drop-off system is suitable for products with high residual value and low MVT (white brand appliances); C3 is used under a drop-off regime to collect EoU products with low residual value and low MVT (e.g., tyres).

\section{Reprocessing options}

The reprocessing stage is the central element of a reverse logistics network, as it is the phase where the economic value of EoU products is recovered using one of the following options:

$\begin{array}{ll}\text { R1 } & \text { Reuse } \\ \text { R2 } & \text { Remanufacturing } \\ \text { R3 } & \text { Recycling }\end{array}$


This classification of reprocessing options can help to establish a relationship with the collection options $(\mathrm{C} 1, \mathrm{C} 2, \mathrm{C} 3)$, providing an integrated perspective of the reverse flow of the SC. Option R1 implies very simple reconditioning operations of EoU products and their reintroduction in the market in similar conditions to those of new products. R2 requires additional work to provide EoU products with similar technical and quality characteristics to their original counterparts. R3 is aimed at recovering the economic value of raw materials of EoU products.

In general, it is impossible to establish a univocal correspondence between collection and reprocessing options, although in most cases recycling (R3) is associated with collection systems through $3 \mathrm{PL}$ or the so-called collective management systems, e.g. packaging material. R2 can be related to several collection options (Savaskan et al., 2004). Thus, EoU products can be collected directly by the manufacturer (C1), as in the case of companies such as Xerox or Samsung, through retailers (C2) as in the case of single-use cameras or mobile phones, or using $3 \mathrm{PL}(\mathrm{C} 3)$ for certain EoU products in the automobile industry. Regarding R1, different collection options are usually employed.

\subsubsection{Vertices and types of vertices in the m-graph}

The representation of a class must identify its inputs and outputs, as well as all resources relevant to SC design.

As mentioned previously, these classes may be of different types, according to the relationships between inputs and outputs. For some types, quantitative relationships between inputs and outputs will be required.

Figure 3 shows suggested representations for all types of classes.

Figure 3.Types of vertices in the m-graph

Type Ta has just one output, which may be the input of one or more recipes, and no incoming arcs. There is at least one Ta in every m-graph.

Type T $\omega$, symmetrical to Ta, has inputs that come from one or more recipes but no outgoing arcs. The vertices of this type may correspond to blocks $U$ and $D$ of the $M$ graph.

Therefore, all the other types of vertices, designated here as $\mathrm{Tg}$, have inputs and outputs. A recipe may receive one or more inputs, which may come from one or more recipes. It can also provide one or more outputs, and each can be the input of one or more recipes.

In order to avoid ambiguities, the use of auxiliary vertices is recommended. These auxiliary vertices gather inputs from different recipes and distribute outputs among several recipes (Figure 3).

In every recipe, we can consider a main vertex that has some information (maybe not perfectly quantified) about the relationship between inputs and outputs. This relationship can correspond to one of the following modes:

Mc A number of units belonging to the elements of a set of (convergence) components generate one unit of final or intermediate product.

Mx Many products are obtained by mixing different raw 
(flexible mix) materials. When its composition is defined by intervals or the recipe ingredients can be selected from a set of alternative raw materials or both, we assign this recipe to mode $\mathrm{Mx}$.
Md
A unit of input provides certain quantities of several outputs. (divergence)
$\mathrm{Mm}$
Some quantities from several inputs provide some quantities (many to many) of several outputs.
Ms
There is no transformation process. The same input coming (sum) from different recipes becomes output. Except for losses, the quantity of output is the total sum of the inputs considered.
$\mathrm{Me}$ (evolution)
A certain proportion of an input becomes an output of a different nature or condition requiring a significant amount of time, either deterministic or random.

If there are alternative recipes for one activity of the process, they may be included in the same m-graph, inscribing them in a common rectangle. However, this will complicate the formulation of the corresponding mathematical model (see 3.4). If there are many steps with alternative recipes or, even more, completely alternative processes, it will be better to formulate a model for each alternative.

\subsection{Stage 4: definition of SC microstructure}

The definition of SC microstructure is aimed at determining the facilities where recipes will be developed, the volume of outcoming and incoming flows between facilities, and the respective transportation means.

The tools for defining the microstructure are the $\mu$-graph(s) and the mathematical programming model(s) based on them. Every m-graph defined at stage 3 generates a $\mu$-graph that provides a particular SC configuration, probably with the help of a mathematical programming or simulation model.

Note where is the place of the mathematical programming models in our approach. When formulating a mathematical programming model, some options are assumed and others are discarded. However, it should be avoided to do this without careful consideration. Moreover, in spite of algorithmic and software developments, it must not be lost of sight that the mixed integer programming problem is NP-hard and this implies that the complexity these models can deal with is bounded.

\subsubsection{The $\mu$-graph}

The $\mu$-graph is based on the m-graph. Every cluster of vertices belonging to a recipe in the m-graph must be replaced with a set of clusters, whose elements are referred to an existing or potential facility where recipes are to be developed.

Once a list of facilities is established, it is advisable to evaluate each one with the aim of retaining only those fulfilling a set of predefined conditions. So, the complexity of the design process is kept within reasonable limits.

The data concerning a facility in a vertex of the $\mu$-graph, relevant for the design of the SC, are: 
- location,

- ownership,

- availability (committed or potential) during the time horizon,

- quantified relationship between inputs and outputs,

- time lag between inputs and outputs,

- output unit cost,

- capacity,

- energy consumption and emissions,

- fixed costs per period,

- opening and closing costs.

Not all these data are required for any facility. For instance, if the owner is a supplier, the leading entity is not concerned with the possible opening or closing costs.

The ownership and the availability during the time horizon are especially important in order to check the consistency between the options adopted in stage 3 and the set of facilities taken into account in the $\mu$-graph, and later to help formulating the mathematical programming model. To this end, it would be useful to assign to each facility a code indicating whether it is owned by the leading entity or by suppliers, when the facility will be open or may be open, and when it will be closed or may be closed.

For instance, if $\mathrm{P} 1$ is adopted for a vertex of the m-graph, all the corresponding facilities in the $\mu$-graph must be owned by the leading entity and, at every period, the joint capacity of the available facilities has to be not less than the required one. On the other hand, if P2, P3 or P4 are adopted, all the corresponding facilities in the $\mu$-graph must be owned by suppliers.

Regarding the clusters of block $U$ in the M-graph, the term "facility" should be replaced with "market" and the capacity will be equivalent to the demand (a numerical value for each period).

If several recipes can be implemented in the same facility, the links between groups of vertices are the same as those in the m-graph. However, the links between groups of vertices related to different facilities generally require new vertices belonging to a new mode: Mt (transportation mode). Any Mt vertex represents a transportation means between two given locations.

For each Mt, we should know:

- delay between delivery and reception,

- fixed and unit transportation costs,

- capacity,

- energy consumption and emissions, and

- investment costs.

Therefore, Mt vertices can be found in the $\mu$-graph but not in the m-graph.

\subsubsection{The models}

In order to optimise the decisions that configure the SC, a mathematical programming model can be derived from the $\mu$-graph. The own structure of the graph and the associated information facilitate the formulation of the model. The decision variables represent flows between facilities or the opening or closing of facilities (concerning 
these latter decisions, the code that is suggested in Section 3.4.1 would permit to determine immediately the sets of the corresponding binary variables). The constraints will define the flow balance in every vertex, capacity limitations and probably financial considerations. For long-lasting production or transportation processes, time differences between the reception of the inputs and the delivering of outputs require consideration.

If product prices are given and market demand can be forecast, a MILP model can be formulated and solved using commercial software or, depending on the complexity of the $\mu$-graph, by using heuristic procedures.

It is worth noting that a model is useful not only in working out the optimal or good solutions, but also in evaluating its sensitivity in different scenarios.

\subsection{Stage 5: Choosing a SC configuration and defining an incident protocol}

The process results in the choice of a SC configuration, according to the established criteria and the adopted process of decision-making.

The final step consists in formulating a strategic plan to deal with incidents and disruptions in the SC effectively, including a description of means to detect incidents and of corrective actions (Tang, 2006; Klibi et al., 2010; Vlajic et al., 2012). Some metrics, such as the ones proposed by SCOR, can help to make the best suitable decisions.

\section{Illustrative example}

An example based on a real situation is described in order to provide insights about how the proposed approach works. This case represents the process of developing a SC for the manufacturing and distribution of an ecological product called ECOXY, an epoxy resin, employed in the repair and maintenance of many different materials, for example boats, surfboards, snowboards, skis, etc. In this case, a European entrepreneur was granted an exclusive license to manufacture and distribute ECOXY in Europe. To this aim, our entrepreneur created the company EUROXY located in a plant close to Barcelona. Now, s/he wants to plan the future configuration of EUROXY SC considering that good market conditions for this product are expected in the next years.

ECOXY consists of two components $A$ and $B$ that can be combined in different ways to obtain different properties and qualities. Component $A$ is obtained as a result of a reaction of a concentrate and an additive. The concentrate is exclusively manufactured and supplied by the licensor in drums of $200 \mathrm{~kg}$. The additive can be bought in the market. On the other hand, component $B$ can be bought to the licensor or manufactured by EUROXY using materials that can be provided by local suppliers.

\section{Stage 1. Object, environment and objectives}

- Object: The first step is to decide between make to stock or customize the product. Taking into account that each one of the multiple applications of ECOXY requires ideally a different composition and that the customisation would give the product a unique character in front of the competitors, the 
entrepreneur decides to customize the product. Therefore, EPOXY will be manufactured to order and marketed online via the Internet. The market includes manufacturers of the sportive equipments indicated above and, based on the ecological attributes of ECOXY, individuals practicing sports in contact with nature and concerned about environmental issues.

- Environment: A significant increase of demand for ECOXY is expected, so the entrepreneur has defined several scenarios for demand (i.e., the demand forecast for each one of the considered commercial areas) and economic conditions, including the evolution of the financial markets.

- Objectives: Since this is a novel product, in the introduction and growth phases, the goal is to settle in the market, as soon as possible, in order to avoid the entry of competitors while minimizing the risk of failure.

\section{Stage 2. Definition of SC macrostructure}

The product cannot be recovered. The drums containing the concentrate will not be returned to the licensor because of the high transportation costs. The packaging material will not be recovered after consumption due to its low residual value and the huge collection costs. However, customers close to the EUROXY's plant/s can return the containers and obtain a discount, if they pick the product up directly at EUROXY.

Therefore, besides the blocks $P$ and $U$, the M-graph will include a block $D$ in order to dispose of drums and other packaging materials and a block $\mathrm{R}$ corresponding to the return of the containers.

\section{Stage 3. Definition of the SC mesostructure}

Structure and production process: The structure of the product has been described at the beginning of this section 4 . Components $A$ and $B$ will be mixed by customer according to the required specifications; therefore, both components will be canned separately and then bundled together to be dispatched to the client

- Options: Concerning A, the concentrate must be bought to PRO (P2 option) and the additive will be bought in the market (P5). Given the conditions of the license, the only option for manufacturing $A$ is $P 1$ (i.e. it has to be manufactured by EUROXY). Component B could be purchased from PRO, but the cost would be greater than manufacturing costs at EUROXY plant/s; so, P1 will be the chosen option. Therefore, it will be necessary to select the suppliers of the corresponding ingredients (P3 option). The packaging materials will be purchased in the market (P5). Regarding the distribution to individual customers, couriers will deliver the product at home (D1). Companies can choose between receiving the product in their facilities (D1) or picking it up from EUROXY (D5), with a discount. Regarding the collection options, EUROXY collects the packaging materials from customers close to facilities (C1). The companies who fetch ECOXY at the EUROXY's warehouses and return the containers can be considered as a special case of $\mathrm{C} 2$; for the reprocessing options, the containers will be reused (R1).

- Specification of modes: The processes to obtain A and B correspond to a convergence mode (Mc). As both can be distributed in containers of different capacities, a divergence mode $(\mathrm{Md})$ is considered for the packing. The final bundle is obtained following a convergence mode (Mc). 
- List of facilities: the entrepreneur must establish a list of possible locations for plants and can associate to each of them a set of suppliers for ingredients and packaging materials and a depot. Once the selected set of locations is obtained, one can pass from the vertices of the m-graph to vertices of the $\mu$ graph.

- Sources of demand: they are the commercial areas used in the definition of the environment in stage 1.

- Means of transport: the use of ships to transport components of $A$ is obliged, as the air transport is too expensive. Therefore, a long period of time is needed to move the materials from U.S. to European ports. At this stage, several alternatives of transportation are also considered and evaluated: from ports and suppliers to plants, from plants to customers.

- $\mu$-graph: the vertices of this graph correspond to the selected potential locations of the plants, and to the means of transport from PRO to the plants and between the plants and the sources of demand.

- Mathematical model: as a result of the decisions that have been adopted throughout the design process, ultimately the decisions to be modelled are: where, when and with which capacity the plants are opened (or expanded), the allocation of each commercial area to each plant and the means of transport, over time, according to the objectives set in stage 1. The remaining decisions (suppliers and depots) will be taken accordingly.

\section{Stage 5. Choose of SC configuration}

The final configuration of the supply chain is given by the resolution of the model. The robustness of the SC must be ensured by means of inventory of the ingredients, giving especial consideration to the concentrate.

\section{Conclusions and future lines of research}

In order to systematise the process of SC design, a top-down hierarchical method called SCOP (Supply Chain Outline Process) has been proposed. The method consists of five stages, including lists of alternatives and guidelines for selecting them and terminology and tools to facilitate communication and coherence analysis.

SCOP is a holistic frame to drive the decision making process of designing the SC. The method forces to consider all the relevant decisions and at every stage only takes into account the pertinent decisions. Given that the elimination of an alternative at one stage implies generally that of many more ones at subsequent stages, the top-down approach simplifies the design process, reducing the number of alternatives to be analysed and requiring the data as they are becoming necessary. This avoids the gathering of data that at the end of the day reveal themselves superfluous.

Particularly, the layout of the method and its considerations show that, contrary to that is assumed, explicitly or not, in many publications, the use of a mathematical programming model is an important step in the process of SC design, but it has not to 
be confused with the whole method. If this confusion prevails, it is very likely that many alternatives will be discarded from the outset, probably without due consideration.

Our prospects of future research include the application of SCOP to complex real SCs, which would help to develop the method itself.

However, the main line of work will be the definition of rules to formulate mathematical models from the information provided by the $\mu$-graph. We think that the terminology and the conditions of coherence included in this paper are a suitable point of depart to tackle this task and that its result will be useful for academics (both for research and teaching) and practitioners as well.

\section{References}

Akçali, E., Çetinkaya, S.,Üster, H. (2009) Network Design for Reverse and ClosedLoop Supply Chains: An Annotated Bibliography of Models and Solution Approaches. Networks, 53, 231-248.

Alumur, S. A., Nickel, S., Saldanha-da-Gama, F., Verter, V. (2012) Multi-period reverse logistics network design. European Journal of Operational Research, 220, 67-78.

Appelqvist, P. Lehtonen, J.M., Kokkonen, J. (2004) Modeling supply chain design: literature survey and case study. Journal of Manufacturing Technology Management, 17, 675-686.

Araújo, D., de Oliveira, T. K., Leite, M. S. A. (2014) Supply chain risk classification: discussion and proposal. International Journal of Production Research, DOI: 101080/00207543.2014.910620.

Balinski, M. (1965) Integer programming: methods, uses, computation. Management Science, 12, 253-313.

Beamon, B. M. (1998) Supply chain design and analysis: models and methods. International Journal of Production Economics, 55, 281-294.

Blackburn, J. D., Guide, V. D. R. J. R., Souza, G. C., \& Van Wassenhove, L. N. (2004) Reverse supply chains for commercial returns. California Management Review, 46, 2, 6-22.

Carle, M. A., Martel, A., Zufferey, N. (2012) The CAT metaheuristic for the solution of multi-period activity-based supply chain network design problems. International Journal of Production Economics, 139, 664-677.

Chandra, C., Grabis, J. (2007) Supply Chain Configuration. Concepts, Solutions, and Applications. Springer.

Chiu, M, Okudan, G. (2011) An integrative methodology for product and supply chain design decisions at the product design stage. Journal of Mechanical Design, 133, 021008.1- 021008.15.

Chopra, S., Meindl, P. (2012) Supply chain management. Strategy, planning and operation, 5th ed. Pearson, Prentice-Hall.

Corominas, A. (2013) Supply chains: What they are and the new problems they raise. International Journal of Production Research, 51, 6828-6835. 
Dotoli, M., Fanti, M. P., Meloni, C., Zhou, M. C. (2005) A multi-level approach for network design of integrated supply chains. International Journal of Production Research, 43, 4267-4287.

Fisher, M.L. (1997) What is the right supply chain for your product? Harvard Business Review, 75, 105-116

Geoffrion, A. M., Graves, G. W. (1974) Multicommodity distribution system design by Benders decomposition. Management Science, 20, 822-844.

Goetschalckx, M., Vidal, C. J., Dogan, K. (2002) Modeling and design of global logistics systems: A review of integrated strategic and tactical models and design algorithms. European Journal of Operational Research, 143, 1-18.

Inman, R. R., Blumenfeld, D. E. (2014) Product complexity and supply chain design. International Journal of Production Research, 52, 1956-1969.

Klibi, W., Martel, A. (2012) Scenario-based Supply Chain Network risk modelling. European Journal of Operational Research, 223, 644-658.

Klibi, W., Martel, A., Guitouni, A. (2010) The design of robust value-creating supply chain networks: A critical review. European Journal of Operational Research, 203, 283293.

Laínez, J. M., Kopanos, G., Espuña, A., Puigjaner, L. (2009) Flexible Design-Planning of Supply Chain Networks. American Institute of Chemical Engineers Journal, 55, 1736-1753.

Lakhal, S., Martel, A., Oral, M., Montreuil, B. (1999) Network companies and competitiveness: A framework for analysis. European Journal of Operational Research, 118, 278-294.

Lakhal, S., Martel, A., Kettani, O., Oral, M. (2001) On the optimization of supply chain networking decisions. European Journal of Operational Research, 129, 259-270.

Lambert, S., Riopel, D., Abdul-Kader, W. (2011) A reverse logistics decision conceptual framework. Computers and Industrial Engineering, 61, 561-581.

Lamothe, J., Hadj-Hamou, K., Aldanondo, M. (2006) An optimization model for selecting a product family and designing its supply chain. European Journal of Operational Research, 169, 1030-1047.

Langlois, P. L., Robertson, R. N. (1992) Networks of innovation in a modular system: Lessons from the microcomputer and stereo component industries. Research Policy 21, 297-313.

Leukel, J. Sugumaran, V. (2013) Formal correctness of supply chain design. Decision Support Systems, 56, 288-299.

Long, Q. (2014) Distributed supply chain network modelling and simulation: integration of agent-based distributed simulation and improved SCOR model. International Journal of Production Research DOI: 10.1080/00207543.2014.910623.

Marsillac, E., Roh, J. J. (2014) Connecting product design, process and supply chain decisions to strengthen global supply chain capabilities. International Journal of Production Economics, 147, 317-329.

M'Barek, W., Martel, A., D'Amours, S. (2010) Designing Multinational Value-Creating Supply Chain Networks for the Process Industry. Working Paper, CIRRELT, CIRRELT2010-51. 
Meixell, M.J, Gargeya, V.B. (2005) Global supply chain design: A literature review and critique. Transportation Research Part E, 41, 531-550.

Melnyk, S. A., Narasimhan, R., DeCampos, H. A. (2014) Supply chain design: issues, challenges, frameworks and solutions. International Journal of Production Research, 52, 1887-1896.

Melo, M. T., Nickel, S., Saldanha da Gama, F. (2005) Dynamic multi-commodity capacitated facility location: a mathematical modeling framework for strategic supply chain planning. Computers \& Operations Research, 33, 181-208.

Micheli, G. J. L., Mogre, R., Perego, A. (2014) How to choose mitigation measures for supply chains, International Journal of Production Research, 52, 117-129.

Ramachandran, K., Krishnan, V. (2008) Design architecture and introduction timing for rapidly improving industrial products. Manufacturing and Service Operations Management, 10, 149-171.

Santoso, T., Ahmed, S., Goetschalckx, M., Shapiro, A. (2005) A stochastic programming approach for supply chain network design under uncertainty. European Journal of Operational Research, 167, 96-115.

Savaskan, R. C., Bhattacharya, S., Van Wassenhove, L. N. (2004) Closed-loop supply chain models with product remanufacturing. Management Science, 50, 2, 239-252.

Seuring, S. (2009) The product-relationship-matrix as framework for strategic supply chain design based on operations theory. International Journal of Production Economics, 120, 221-232.

Sha, D.Y., Che, Z.H. (2006) Supply chain network design: partner selection an production/distribution planning using a systematic model. Journal of the Operational Research Society, 57, 52-62.

Shen, Z. J. M. (2007) Integrated supply chain design models: a survey and future research directions. Journal of Industrial and Management Optimization, 3, 1-27.

Simchi-Levi, D., Kaminski, P., Simchi-Levi, E. (2008) Designing and Managing the Supply Chain. Concepts, Strategies \& Case Studies. Irwim McGraw-Hill.

Stadtler, H. (2002). Supply Chain Management - An Overview. In Stadtler, H.; Kilger, C. Supply Chain Management and Advanced Planning. Springer.

Stadtler, H., Kilger, C. (2005) Supply Chain Management and Advanced Planning. Concepts, Models, Software and Case Studies. Springer Berlin: Heidelberg.

Stock, J. R., Boyer, S. L. (2009) Developing a consensus definition of supply chain management: a qualitative study. International Journal of Physical Distribution and Logistics Management, 39, 690-711.

Talluri, S., Baker, R. C. (2002) A multi-phase mathematical programming approach for effective supply chain design. European Journal of Operational Research, 141, 544558.

Tang, C. S. (2006) Robust Strategies for Mitigating Supply Chain Disruptions, International Journal of logistics: Research and Applications, 9, 1, 33-45.

Tang, O., Musa, S.N. (2011) Identifying risk issues and research advancements in supply chain risk management, International Journal of Production Economics, 133, 25-34.

Ülkü, S., Schmidt, G. (2011) Matching Product Architecture with Supply Chain Configuration. Production and Operations Management, 9, 225-241. 
Ulrich, K. (1995) The role of product architecture in the manufacturing firm. Research Policy, 24, 419-440.

Vila, D., Martel, A., Beauregard, R. (2006) Designing logistics networks in divergent process industries: A methodology and its application to lumber industry. International Journal of Production Economics, 102, 358-378.

Viswanadham, N., Kameshwaran, S. (2013) Ecosystem-aware Global Supply Chain Management. World Scientific.

Vlajic, J. V, van der Vorst, J. G. A. J., Haijema, R. (2012) A framework for designing robust food supply chains. International Journal of Production Economics, 137, 176189.

Vonderembse, M. A., Mohit, U., Huangc, S. H., Dismukesd, J. P. (2006) Designing supply chains: Towards theory development. International Journal of Production Economics, 100, 223-238.

Wagner, S. M., Neshat, N. (2010) Assessing the vulnerability of supply chains using graph theory. International Journal of Production Economics, 126, 121-129.

Wang, G., Huang, S.H., Dismukes, J.P. (2004) Product-driven supply chain selection using integrated multi-criteria decision-making methodology. International Journal of Production Economics, 91, 1-15

Figure 1.General layout of SCOP

Figure 2. Examples of M-graphs

Figure 3.Types of vertices in the m-graph 
General layout of the SCD $254 \times 190 \mathrm{~mm}(96 \times 96 \mathrm{DPI})$

Stage 1: Object, environment and objectives

- Definition of the SC object

Analysis of the environment (scenarios and potential markets)

- Determination of the objectives.

Stage 2: Definition of SC macrostructure

- Identification of activity blocks and relation between them

- Analysis of recovery options

Stage 3 Definition of SC mesostructure

- Definition of the product structure and production process

- Options for each SC activity or group of activities

- Specification of a mode for each main vertex of every recipe

- m-graph

Stage 4: Definition of SC microstructure

- List of facilities where the recipes can be done

- Sources of demand

- Means of transport between facilities or facilities and sources of demand

- $\mu$-graph

- Mathematical model

Stage 5 : choose of SC configuration

- Selection of SC configuration

- Definition of protocols in case of incidents

- Implementation of the SC 


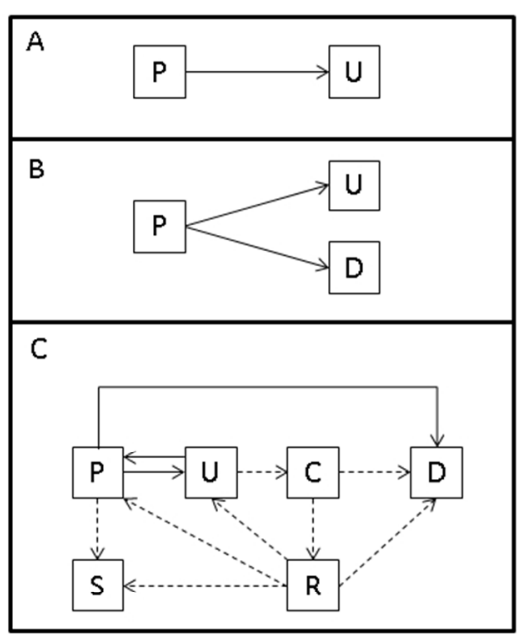

Examples of M-graphs $254 \times 190 \mathrm{~mm}(96 \times 96 \mathrm{DPI})$

http://mc.manuscriptcentral.com/tprs Email: ijpr@tandf.co.uk 


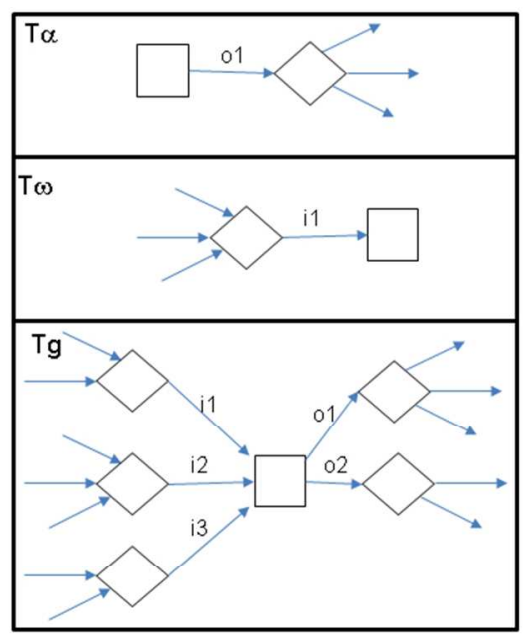

Types of vertices in the m-graph $254 \times 190 \mathrm{~mm}(96 \times 96 \mathrm{DPI})$ 
Reviewer: 1

Thank you for your prompt and constructive review. According to it, we have prepared a revised version of the paper. The changes have been coloured in yellow.

Comments to the Author

The first half of the paper has improved significantly. However, there are major issues that have to be improved in the almost completely rewritten sections in the second half of the paper. Please note that I am referring to the manuscript pages and not to the PDF page numbering.

_Minor issues:

Page 4 line 39-41: There is something wrong with the sentence:

"... our analysis reveals that there is not in it any sufficiently detailed, complete ..."

Maybe the authors mean: "... our analysis reveals that there is no sufficiently detailed, complete ..."

The sentence has been rewritten (page 4) according to your suggestion.

Page 4: Section 3 heading. The name of the method SCD is a bit too generic. Maybe the authors could find a more specific name for their approach. Otherwise, the reader could think that the characteristics mentioned refer to supply chain design in general and do not specifically refer to their approach.

We appreciate your suggestion. We have renamed our approach. SCD has been replaced with SCOP (Supply Chain Outline Process) in all the paper. The new denomination is introduced in page 4.

Page 5 point e: The bullets a. to $d$. are very clear to the reader and it is understandable why holistic SCD should satisfy those characteristics. Point e. is very specific and is not clearly formulated as a characteristic. I would propose to rephrase point e.

We agree with your comment. Point e has been rephrased (page 5).

Page 5 point i: This does not seem to be a characteristic but rather a remark. Otherwise, the authors should clarify this characteristic. 
We also agree. Point $\mathrm{i}$ has been deleted, since the main idea of this point is presented in other parts of the paper. Accordingly, the former point $\mathrm{j}$ is now point $\mathrm{i}$ (page 5).

Page 6: "It is not always necessary to draw the graphs (M-graph, m-graph and $\mu$-graph) mentioned." However, the names in the brackets are mentioned for the first time. They should be defined beforehand. Moreover, in the following pages the authors always refer to the end of section 3 where the graphs are defined.

This paragraph has been rewritten. Now, we mention it in Fig. 1, in which the graphs appear for the first time in the paper.

Page 14: The first half of the first sentence of section 4 does not seem to be correct.

Your observation has been taken into account when we have completely rewritten section 4, according to your comment about it.

\section{_Major issues:_}

Page 12-13 (Section 3.4.1.): Why do the authors introduce a mathematical notation if it is not used afterwards? The new formulated section does not enhance the readability of the article and it does not help to understand the meaning and purpose of this graph. Moreover, the authors have to improve the language in this section.

This section has been rewritten in order to reduce its length and to eliminate superfluous details. In the previous version we have tried to illustrate the possibility of defining a notation in the frame of the approach. However, most likely this can be suggested, as we have tried to do in the new version, without introducing so many details as in the previous one.

Section 4 in general: This section presents the end result of the approach but it does not show how each step itself is conducted. The example does not clearly show the benefit(s) of the approach. I do not see that the decision maker is supported by this approach. I still do not see the benefit of the graphical representations of your different kinds of graphs. The authors have to improve the language in this section.

We have rewritten section 4 with the purpose of trying to show how the proposed approach may help the decision maker both to not forget the consideration of any relevant decision and to rule out progressively alternatives that a priori might seem appropriate. To this aim, now the section begins depict the starting situation, to proceed thereafter to describe synthetically the decisions adopted progressively in the design process and how the approach really helps the entrepreneur. We hope that the considerations concerning the role of the graphs and their graphical 
representation in the whole approach may also help to clarify their role in the example.

Section 5: The contribution of the paper is not clearly presented in section 5. If I would summarize the contribution which the authors have expressed in section 5, in simple terms, it would be the following: It seems that the approach leads to a rough and quick but rather holistic picture of a supply chain. Therefore, the approach neglects "unnecessary details" and does not include a mathematical programming model. However, I am convinced that this is not the author's intended contribution.

Really, we are sorry that section 5 in the previous version may be interpreted as you point out. This, obviously, was not our intention. Accordingly, second and third paragraphs of the section have been reformulated (page 15). 
Reviewer: 2

Thank you for your prompt and constructive review. In the new revised version the changes relative to the preceding one have been coloured in yellow.

\section{Comments to the Author}

My observations have been properly addressed, I have no further comment for the work other than consider a SCD simulation or system dynamics prior (probably in the same publication) to real SCs scenarios, that would feed the hypotheses generation and make the work process more interesting.

We have introduced in page 6 a short comment about the use of simulation in the generation of scenarios. 